\title{
Modeling of Response Curves of Chemiresistive Sensors with Modified Gompertz Functions
}

\author{
G. Tang ${ }^{1}$, Sachin T. Navale ${ }^{1}$, P. Yang ${ }^{1}$, Florian J. Stadler ${ }^{1, *}$ \\ ${ }^{1}$ College of Materials Science and Engineering, Nanshan District Key Lab for Biopolymers and Safety \\ Evaluation, Shenzhen University, Shenzhen-518060, P. R. China \\ *E-mail: fjstadler@szu.edu.cn
}

\begin{abstract}
:
A large multitude of chemiresistive gas sensors have been proposed to detect minute amounts of toxic or noxious gases using inexpensive setups. While the ultimate value of the response of gas sensors is well-established for analyzing the characteristics so far has been mostly neglected or given a lower priority. In this communication, we show that the time-dependent response can be well modeled by slightly modified Gompertz functions and that the derived parameters can be correlated with the detection mechanism, making it a useful additional analysis method for a better understanding of gas sensing. These new methods in the analysis of the gas sensing data will be useful in two different fields. Firstly, it allows for the analysis of acquired data in order to get a deeper understanding of e.g. differences between different gas sensors/gases by being finally able to compare easily determinable and scientifically meaningful parameters. Secondly, the fact that the sensor response can be easily modeled by three parameters, each of which have their correlations with gas concentration, makes it easy to completely parameterize the sensor response, which in turn lends itself for applying these sensors practically, as the parameterized response can be used for determining the true gas concentration from the time dependent response. This could be used for speeding up the gas sensor's alarm triggering for hazardous gas concentrations.
\end{abstract}

Key words: Metal oxides, Sensing properties, Response curves, Modeling, Gompertz functions 